\title{
Camas de Combustibles forestales y carbono en México
}

\section{Forest fuel beds and carbon in Mexico}

\author{
J. Germán Flores-Garnical", Julio C. Wong-González² y Fernando Paz-Pellat ${ }^{3}$
} 1 Instituto Nacional de Investigaciones Forestales,
Agrícolas y Pecuarias (INIFAP). Campo Experimental
Centro Altos de Jalisco. Tepatitlán de Morelos, Jalisco,
México.

\author{
2 Programa Mexicano del Carbono. Texcoco, Estado \\ de México, México. \\ 3 Colegio de Postgraduados y Programa Mexicano
}

del Carbono. Texcoco, Estado de México, México.

\section{RESUMEN}

Los combustibles forestales juegan un papel importante en el ámbito del calentamiento global, por lo que la adecuada estimación de las camas de combustible permitirá disminuir los niveles de incertidumbre de los contenidos de carbono (C) de los ecosistemas forestales. Este trabajo describe los procesos de evaluación de las cargas de mantillo (hojarasca y capa de fermentación, M) y de material leñoso caído (MLC); además, presenta una síntesis de los contenidos de C registrados en México. La evaluación de M y MLC se basó principalmente en la estrategia de intersecciones planares. Los contenidos de $\mathrm{C}$ se determinaron usando diferentes porcentajes de contenido de $\mathrm{C}$ respecto a la masa (peso seco), que variaron desde $47.5 \%$ hasta $66.9 \%$ aunque, en general, se consideró $50 \%$. La mayoría de trabajos revisados se enfocaron en ecosistemas de regiones templadas, con registros de $9.43 \mathrm{Mg} \mathrm{C}$ ha $^{-1}$ de MLC, mientras que para selvas se registraron $8 \mathrm{Mg} \mathrm{C} \mathrm{ha}^{-1}$. En cuanto a $\mathrm{M}$, se observó $6.91 \mathrm{Mg} \mathrm{C} \mathrm{ha}^{-1}$ y $4.25 \mathrm{Mg} \mathrm{C}^{-1}$ en la capa de hojarasca y $5.88 \mathrm{Mg} \mathrm{C}^{-1}$ y $1.39 \mathrm{Mg} \mathrm{C} \mathrm{ha}^{-1}$ en la capa de fermentación para regiones templadas y selvas, respectivamente. Se encontró muy poca información sobre las evaluaciones de C correspondientes a M y MLC, la mayoría enfocada a ecosistemas templados y tropicales, por lo que se resalta la necesidad de considerar otras regiones climáticas. Las evaluaciones de las cargas de los combustibles forestales deben hacerse en forma estandarizada y con protocolos comunes, de tal forma que la información generada pueda ser tanto comparable como compatible. Esto permitirá reducir los niveles de incertidumbre y presentar la información en diferentes escalas. De esta manera, será posible no solo reducir los niveles de incertidumbre, sino también escalar la información.

PALABRAS ClAVE: almacenamiento de carbono, bosque templado, bosque tropical, capa de fermentación, hojarasca, material leñoso caído.

\section{ABSTRACT}

Forest fuels play an important role in the field of global warming, therefore a proper estimation will allow a decrease of the uncertainty levels of carbon $(\mathrm{C})$ contents. This paper describes the evaluation process for loads of duff (litter and fermentation layer, M) and downed woody material (MLC). A summary of the $\mathrm{C}$ content reported in Mexico is also presented. The evaluation of these fuels has been primarily based on the strategy of planar intersections. The contents of $\mathrm{C}$ were determined using different percentages of $\mathrm{C}$ content with respect to mass (dry weight), ranging from $47.5 \%$ to $66.9 \%$, although a percentage of $50 \%$ was used, in general. Most works has been focused on temperate ecosystems, where $9.43 \mathrm{Mg} \mathrm{C} \mathrm{ha}^{-1}$ of MLC was reported. While for tropical forests there was $8 \mathrm{Mg} \mathrm{C}^{-1}$. For $\mathrm{M}$ was reported 6.91 $\mathrm{Mg} \mathrm{C} \mathrm{ha}^{-1}$ and 4.25 $\mathrm{Mg} \mathrm{C} \mathrm{ha}^{-1}$ in duff, and 5.88 $\mathrm{Mg} \mathrm{C} \mathrm{ha}^{-1}$ and $1.39 \mathrm{Mg} \mathrm{C} \mathrm{ha}^{-1}$ in the fermentation layer for temperate regions and tropical forests, respectively. It is concluded that there is little information on C evaluations regarding $\mathrm{M}$ and $\mathrm{MLC}$. In addition, other regions should be considered and standardized evaluation procedures should be used. In this way, the information generated will be comparable and compatible. This will not only reduce levels of uncertainty, but also present information at different scales.

KEYWORDS: carbon storage, temperate forest, tropical forest, fermentation layer, litter, duff. 


\section{INTRODUCCIÓN}

Los ecosistemas forestales son una fuente de combustibles que determina varios factores relacionados a los incendios forestales (i.e. riesgo, peligro, intensidad, frecuencia, entre otros). Más aun, la masa de combustibles disponibles difiere entre estratos y entre ecosistemas forestales, definiendo, entre otros factores, el tipo de incendio que se presenta (superficial, de copa, subterráneo o mixto) (Maldonado, Paz, Olguín y de Jong, 2012); lo cual, a su vez, condiciona el grado del impacto del incendio. Debido a esto, los combustibles forestales han sido ampliamente estudiados (Morfín, Jardel, Alvarado y Michel, 2012; Nájera y Hernández, 2007; Flores y Omi, 2003), definiéndose una clasificación de los mismos que ha ido variando con base en los avances técnicos. Esto es importante en el ámbito del calentamiento global, ya que el adecuado conocimiento de los combustibles forestales ayuda a determinar con menor incertidumbre los niveles de almacenamiento de carbono (C); lo cual, a su vez, define el potencial de emisiones de $\mathrm{C}$ de los ecosistemas forestales y ayuda a entender mejor los flujos de $\mathrm{C}$ que en ellos ocurren (Maldonado et al., 2012). Aproximadamente 20\% de las emisiones de $\mathrm{C}$ en la atmósfera provienen de quemas de biomasa (Tubiello et al., 2015; Crutzen y Andreae, 1990). Sin embargo, en muchos casos se considera a los combustibles forestales como un solo elemento, por lo que sus evaluaciones, en ocasiones, son demasiado generalizadas o con un alto nivel de incertidumbre (Kurz et al., 2016). Existen diferentes variaciones en el concepto de combustibles forestales, donde, primeramente, se distingue entre combustibles propios de la biomasa y combustibles muertos (necromasa) (Xelhuantzi, Flores y Chávez, 2011). De esta forma, se tienen grupos de combustibles; por ejemplo, arbolado adulto, arbolado joven, material leñoso, troncos y mantillo; que, a su vez, están definidos por varios componentes (ramas, fuste, hierbas, ramillas de $0 \mathrm{~cm}$ a 0.6 $\mathrm{cm}$, trozas $>7.5 \mathrm{~cm}$, entre otros). Dentro de estos grupos de combustibles destacan los que corresponden al mantillo (M) y al material leñoso caído (MLC), ya que estos pueden definir, junto con otros factores, el riesgo y peligro de un incendio forestal. Además, el M y el MLC forman parte de los principales almacenes de $\mathrm{C}$ en las comunidades vegetales (Penman et al., 2003). Debido a lo anterior, el M y el MLC pueden ser una fuente de emisiones de C (Woodale y Williams, 2005), la cual dependerá de la cantidad y calidad de material disponible para quemarse. El MLC comprende toda la madera muerta que se localiza por encima del suelo, tal- como ramas, troncos, tocones y árboles muertos en pie; sin embargo, estos últimos suelen separase en algunas mediciones ya que no están caídos y su método de evaluación es diferente al del material caído. El M considera, básicamente, dos capas: a) hojarasca, que se forma en el piso forestal y está constituido principalmente por hojas y acículas, las cuales están relativamente completas; b) fermentación, consistente en una capa de hojarasca en proceso de descomposición que se encuentra debajo de la capa de hojarasca. Por lo general, las evaluaciones de estos grupos de combustibles se refieren a la masa (carga) que tienen por unidad de superficie del terreno y se expresa generalmente en tonelada por hectárea $\left(\mathrm{Mg} \mathrm{ha}^{-1}\right)$. Con esta información y con base en un porcentaje de contenido de $\mathrm{C}$ respecto a la masa (peso seco) (generalmente $50 \%$ ), se estima el contenido de $\mathrm{C}$ en megagramos por hectárea $\left(\mathrm{Mg} \mathrm{ha}^{-1}\right)$. La madera muerta es considerada una reserva de $\mathrm{C}$ y un sitio para la fijación de nitrógeno y acumulación de materia orgánica; de este modo, actúa como un liberador de fertilizante a largo plazo; sin embargo, continúa siendo materia de debate si la cosecha intensiva de madera puede desabastecer de ciertos nutrimentos a los bosques (Hunter, 1990; Torres, 1994).

La evaluación de las cargas de combustibles y la correspondiente estimación de los contenidos de $\mathrm{C}$ del $\mathrm{M}$ y del MLC son procesos que contemplan varios pasos y técnicas. No obstante, esto no implica que sea complicado, básicamente porque ya se cuenta con técnicas definidas para ambos propósitos. Sin embargo, actualmente es difícil comparar o compartir datos sobre los contenidos de C tanto del M, como del MLC. Esto se debe, esencialmente, a que no se han determinado procesos estandarizados para estos fines; de tal forma que pueda partirse de, por ejemplo, una misma clasificación de regiones climáti- 
cas, tipos de vegetación, procesos de evaluación de cargas de combustibles y clasificación de tipos de combustibles (vivos y muertos). Como resultado de esto, en la mayoría de los casos, se trabaja con base en procesos y clasificaciones diferentes.

\section{Objetivos}

De acuerdo con lo discutido anteriormente, el trabajo tiene como objetivo presentar una revisión de los procesos de medición y estimación de las cargas de mantillo y material leñoso caído y contenidos de $\mathrm{C}$ encontrados en México, principalmente para bosques y selvas.

\section{MATERIALES Y MÉTODOS}

\section{Sistema de clasificación de combustibles}

Para entender mejor el papel que juegan las cargas de $\mathrm{M} \mathrm{y}$ MLC, se debe conocer su ubicación dentro del complejo de combustibles forestales, los cuales comprenden la materia orgánica vegetal (viva o muerta) de los ecosiste-

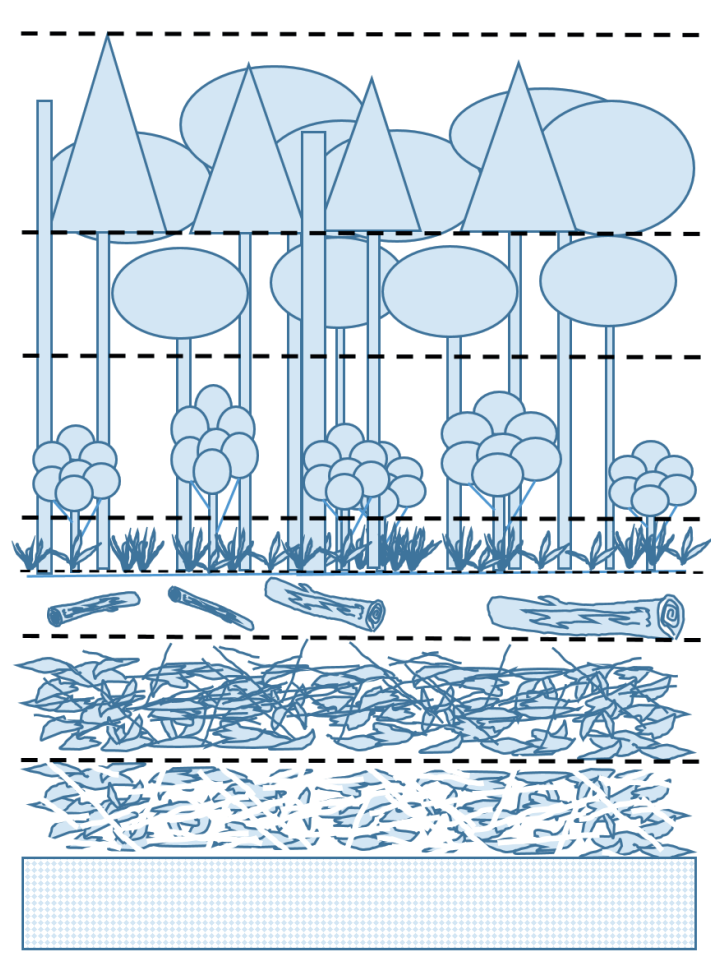

mas terrestres que es susceptible de entrar en combustión. Dentro de estos, se considera follaje, fustes, raíces, troncos, tocones, ramas, hojarasca y capa de fermentación (Flores y Moreno, 2005; Pyne, Andrews y Laven, 1996); los cuales se han clasificado con diferentes propósitos (modelaje, estimación de cargas, simulación, entre otros) (Prichard et al., 2013). Sin embargo, el concepto de camas de combustibles (CaCom) soporta la clasificación más reciente y completa de los combustibles forestales, que se basa en el sistema de clasificación de características de combustibles (FCCS por sus siglas en inglés) propuesto por Ottmar, Sandberg, Riccardi y Prichard (2007). En ese sistema de clasificación, los combustibles se dividen en estratos que incluyen tanto material combustible vivo, como muerto (Fig. 1), por lo puede usarse para una adecuada clasificación de combustibles enfocada en la evaluación de los contenidos de C. Lo anterior permitirá apoyar la definición de un protocolo que acceda a estandarizar la evaluación de $\mathrm{C}$ en diferentes ecosistemas forestales (Barrales et al., 2012).

DOSEL (Copas y troncos en pie)

SUBDOSEL Y COMBUSTIBLES ESCALONADOS

ARBUSTOS

VEGETACION BAJA (Hierbas y pastos))

MATERIAL LEÑOSO CAIDO

HOJARASCA FRESCA

CAPA DE FERMENTACION Y RAICES

(hojarasca y madera en descomposición)

\section{SUELO MINERAL}

Figura 1. Modelo conceptual de los componentes de una cama de combustibles forestales (adaptado de Morfín et al., 2012). 
Al respecto, Cruz, Escalante, González, Guerra y Etchevers (2012) hacen una propuesta de clasificación de combustibles forestales, desde la perspectiva de evaluación de $\mathrm{C}$, que en varios aspectos coincide con la clasificación sugerida por Eggleston, Buendia, Miwa, Ngara y Tanabe (2006) y el Programa Mexicano del Carbono [PMC] (2011), quienes especifican considerar los estratos como almacenes de C. En la tabla 1 se muestran las coincidencias y diferencias de estas dos clasificaciones o esquemas de estimación; aunque ambos casos generalizan la clasificación, integrando varias fuentes de combustibles en unos pocos estratos, lo que puede generar un nivel de incertidumbre alto. Por ejemplo, desde la perspectiva de CaCom y $\mathrm{C}$ de la biomasa aérea $\left(\mathrm{C}_{\mathrm{BA}}\right)$ puede desglosarse en Morfín et al. (2012): dosel, subdosel, arbustos, vegetación baja, material leñoso, hojarasca fresca y capa de fermentación. No obstante, la clasificación de las camas de combustibles puede fragmentarse más aún, con los siguientes enfoques: 1) precisar el cálculo de contenido de C y 2) contar con datos más específicos que determinen un nivel de certidumbre más robusto. De acuerdo con esto, se propone una clasificación que integre la perspectiva de las CaCom, con las alternativas basadas en la evaluación de C. De esta forma, en la integración se definen nueve estratos, con sus respectivos subestratos y componentes (Tabla 1). En esta propuesta se parte de una primera clasificación general, donde se diferencian los combustibles vivos (biomasa) y los combustibles muertos (necromasa), separando en estos últimos a los árboles muertos en pie, que son considerados en las CaCom como parte del dosel.

La propuesta integral (PI) de clasificación de combustibles apoya la definición de un protocolo, donde se establezcan los estándares de evaluación de combustibles, para la estimación de $\mathrm{C}$ en diferentes ecosistemas forestales (Barrales et al., 2012). Es importante señalar que ya existen metodologías específicas para la evaluación de cada componente de combustibles forestales, con lo que, además, se puede evaluar $\mathrm{C}$ a diferentes niveles, de acuerdo con los estratos, subestratos o componentes que se consideren. Más aún, la PI es compatible con la estrategia del Inventario Nacional Forestal y de Suelos (Infys) (Comi- sión Nacional Forestal [Conafor], 2016), en donde se consideran como los principales almacenes de $\mathrm{C}$ a la biomasa viva, material leñoso caído (incluyendo madera muerta en pie), mantillo, capa de fermentación y suelo (Maldonado et al., 2012), que a su vez pueden acoplarse a los almacenes considerados en la Guía de Buenas Prácticas del IPCC (Penman et al., 2003). En esta perspectiva, con base en la información del Infys se han caracterizado y clasificado camas de combustibles prioritarias en México (Chávez et al., 2014). Esta clasificación fue realizada mediante análisis multivariantes y geoespaciales, mientras que la caracterización se realizó mediante técnicas de minería de datos y manejo de bases de datos relacionales. Como resultado se tiene un catálogo nacional de camas de combustibles forestales, estratificado de acuerdo con las diversas regiones bioclimáticas y tipos de cobertura vegetal de México (Chávez et al., 2014).

\section{Evaluación de cargas de combustibles}

Como se ha mencionado, la mayoría de los trabajos sobre evaluación de cargas de MLC y M en México usan adaptaciones a la metodología propuesta por Brown (1974). Para esto, primeramente se debe definir la estrategia de muestreo, con lo cual se determina la ubicación de los sitios (o conglomerado) de muestreo. Los muestreos pueden ser aleatorios o sistemáticos, lo cual depende de los objetivos del trabajo, mientras que el diseño del sitio de muestreo puede variar en forma y tamaño, lo que depende de la heterogeneidad de la vegetación, así como de la topografía del terreno. La figura 2 presenta una versión estilizada del diseño de un sitio de muestreo para evaluar MLC y M que, en general, comprende los siguientes aspectos: a) tres transectos de intersecciones planares. Sobre estos transectos lineales se mide el combustible grueso o material leñoso caído. Cada transecto se marca con cuerdas de una longitud conocida (generalmente $7 \mathrm{~m}$ ), orientadas a $0^{\circ}, 120^{\circ}$ y $240^{\circ}$ de azimut. Los combustibles de $1 \mathrm{~h} \mathrm{y} 10 \mathrm{~h}$ (Tabla 2) se miden en una distancia de $0 \mathrm{~m}$ a $3.5 \mathrm{~m}$, mientras que los de $100 \mathrm{~h}$ y $1000 \mathrm{~h}$ de $0 \mathrm{~m}$ a $7 \mathrm{~m}$, sobre la línea de muestreo; b) un circulo de $3.5 \mathrm{~m}$ de radio. Este círculo, concéntrico, se utiliza para la medición de combustible 


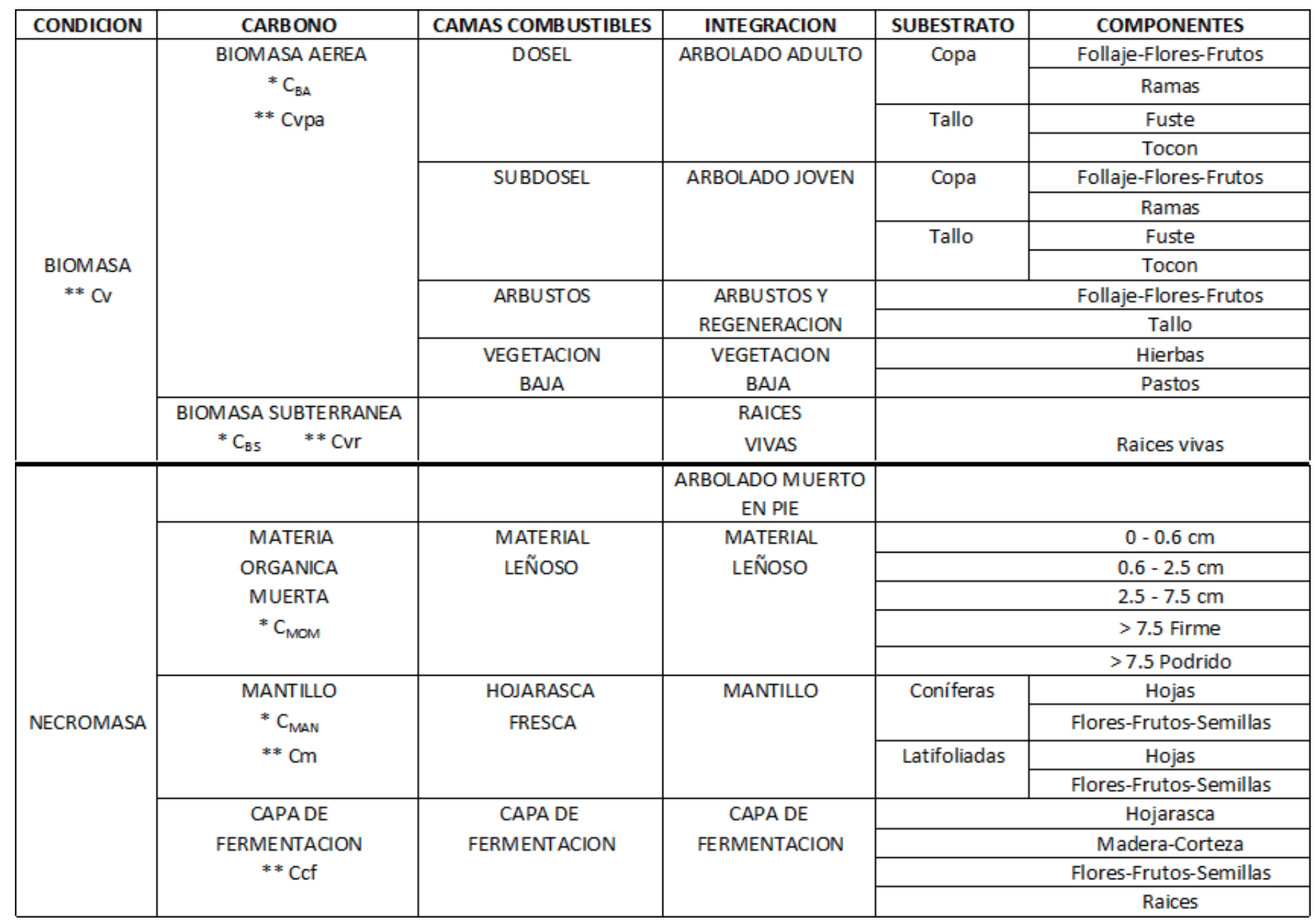

Tabla 1. Comparación de diferentes clasificaciones de combustibles forestales, enfocadas a la evaluación de contenidos de carbono.

Donde: ** Ccf = Carbono en la capa de fermentación; Cm = Carbono en el mantillo; Cv = Carbono en la vegetación; Cvpa = Carbono en la vegetación de la parte aérea; Cvr = Carbono en la vegetación de la parte radicular (Cruz et al., 2012).

${ }^{*} \mathrm{C}_{\mathrm{BA}}=$ Carbono de la biomasa aérea; $\mathrm{C}_{\text {MAN }}=$ Carbono del mantillo; $\mathrm{C}_{\mathrm{BS}}=$ Carbono de la biomasa subterránea; $\mathrm{C}_{\text {Mom }}=$ Carbono de la materia orgánica muerta (Eggleston et al., 2006; Paz y Cuevas, 2012).

vivo, arbustos, hierbas, pastos y regeneración y c) tres cuadros de $30 \mathrm{~cm} \times 30 \mathrm{~cm}$. Se trata de cuadrantes en los que se evalúa profundidad y porcentaje de cobertura de la hojarasca y de mantillo orgánico. Estos se encuentran al final de cada transecto y son utilizados también para la toma de muestras de hojarasca y materia orgánica.

\section{Material Leñoso Caído (MLC)}

De acuerdo con el diámetro que presentan sus elementos, el MLC se clasifica en: fino, regular, mediano y grueso (Tabla 2). Originalmente las categorías fueron definidas en pulgadas, por lo que se muestra la conversión al Sistema Internacional (Brown, 1974; Rodríguez, Hubert, Cíntora, Contreras y de la Rosa, 2011). Otra manera de clasificar a los combustibles es por el tiempo de retardo que presentan (Fosberg y Deeming, 1971), el cual se refiere al tiempo que tardan en perder o ganar aproximadamente $66 \%$ de la diferencia entre el contenido de la humedad inicial y la humedad relativa del aire a cierta temperatura establecida (Simard, 1968). El método más común en la medición del MLC está basado en el muestreo de intersecciones planares lineales, donde se contabiliza la cantidad 


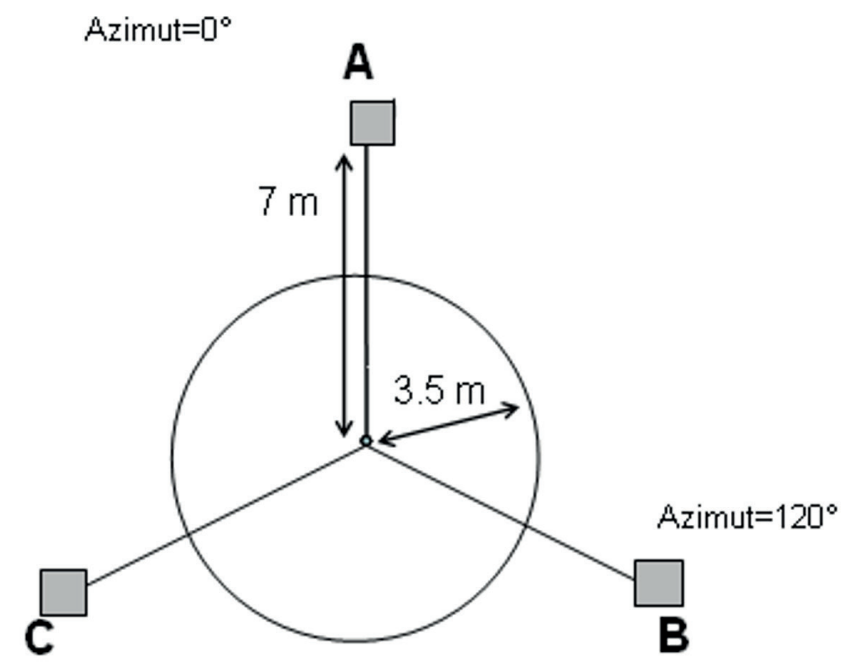

Figura 2. Esquema con el diseño del sitio de muestreo de combustibles forestales, mostrando longitud y orientación de los transectos (A, B y C cuadros de $30 \mathrm{~cm} \times 30 \mathrm{~cm}$ ).

de material leñoso que se encuentra justo por debajo de la línea del transecto. La frecuencia del material se mide de acuerdo con las diferentes categorías de diámetro (Fig. 3). Posteriormente, se estiman las cargas de cada una de estas categorías, con base en una serie de ecuaciones propuestas por Brown, Oberheu y Johnston (1982).

Tabla 2. Clasificación del MLC por diámetro y tiempo de retardo.

\begin{tabular}{ccc}
\hline Categoría & Diámetro $(\mathrm{cm})$ & Tiempo de retardo (horas) \\
\hline Finos & $0-0.5$ & 1 \\
Regulares & $0.51-2.5$ & 10 \\
Medianos & $2.51-7.5$ & 100 \\
Gruesos & $>7.5$ & 1000 firme \\
& $>7.5$ & 1000 podrido \\
\hline
\end{tabular}

Existen varios trabajos que describen la metodología para evaluar el C almacenado del MLC. Tal es el caso de la Guía de Buenas Prácticas del IPCC (Penman et al., 2003). No obstante lo anterior, son pocos los trabajos que se han realizado sobre la estimación exclusiva del C en madera muerta caída (Navarrete et al., 2011). Además, el $\mathrm{C}$ en el M y el MLC puede tener altas fluctuaciones y ser difícil de medir (Navarrete, 2006); no obstante, su evaluación es de gran importancia en proyectos REDD+ (Reducción de Emisiones por Deforestación y Degradación forestal evitadas, más conservación, incremento de almacenes y manejo forestal sustentable), siempre y cuando se genere información con la menor incertidumbre posible, para lo cual es fundamental considerar la densidad específica de cada combustible. A pesar de esto, es frecuente que se calcule el C con la densidad de la madera de otras especies y bajo condiciones ambientales muy distintas a las de México (Navarrete, 2006).

\section{Mantillo (M)}

Una vez que se ha instalado el sitio de muestreo de combustibles y se han ubicado los transectos (por lo general tres), al final de los transectos se coloca un cuadro de 30 $\mathrm{cm} \times 30 \mathrm{~cm}$ para evaluar los combustibles ligeros (Xelhuantzi et al., 2011), los cuales se definen en la figura 4 : a) capa de fermentación: capa en donde se localizan hojarasca, hierbas y pastos en estado de descomposición, o inicio de la misma, tendiendo a la formación de materia orgánica; esta capa también se identifica por la fragmentación de las hojas o acículas; y b) capa de hojarasca: capa donde se localizan las hojas, o acículas, caídas de árboles o arbustos, donde los elementos de esta capa (hojas o acículas) se encuentran completos o fragmentados, pero reconocibles. Una vez ubicado el cuadro de 30 $\mathrm{cm} \times 30 \mathrm{~cm}$, todo el material combustible es colectado y separado en capa de hojarasca y capa de fermentación. En el laboratorio se revisa la separación realizada en campo y se procede a su secado hasta obtener peso constante. Seco el material, se efectúan las estimaciones para determinar las cargas $\left(\mathrm{Mg} \mathrm{ha}^{-1}\right)$ de combustibles correspondientes. Las estimaciones del contenido de $\mathrm{C}$ en el M se han hecho con base en la definición de un porcentaje de contenido de $\mathrm{C}$ respecto a la masa (peso seco), el cual, generalmente fluctúa en valores alrededor de 50\% (Romero, 2008). 

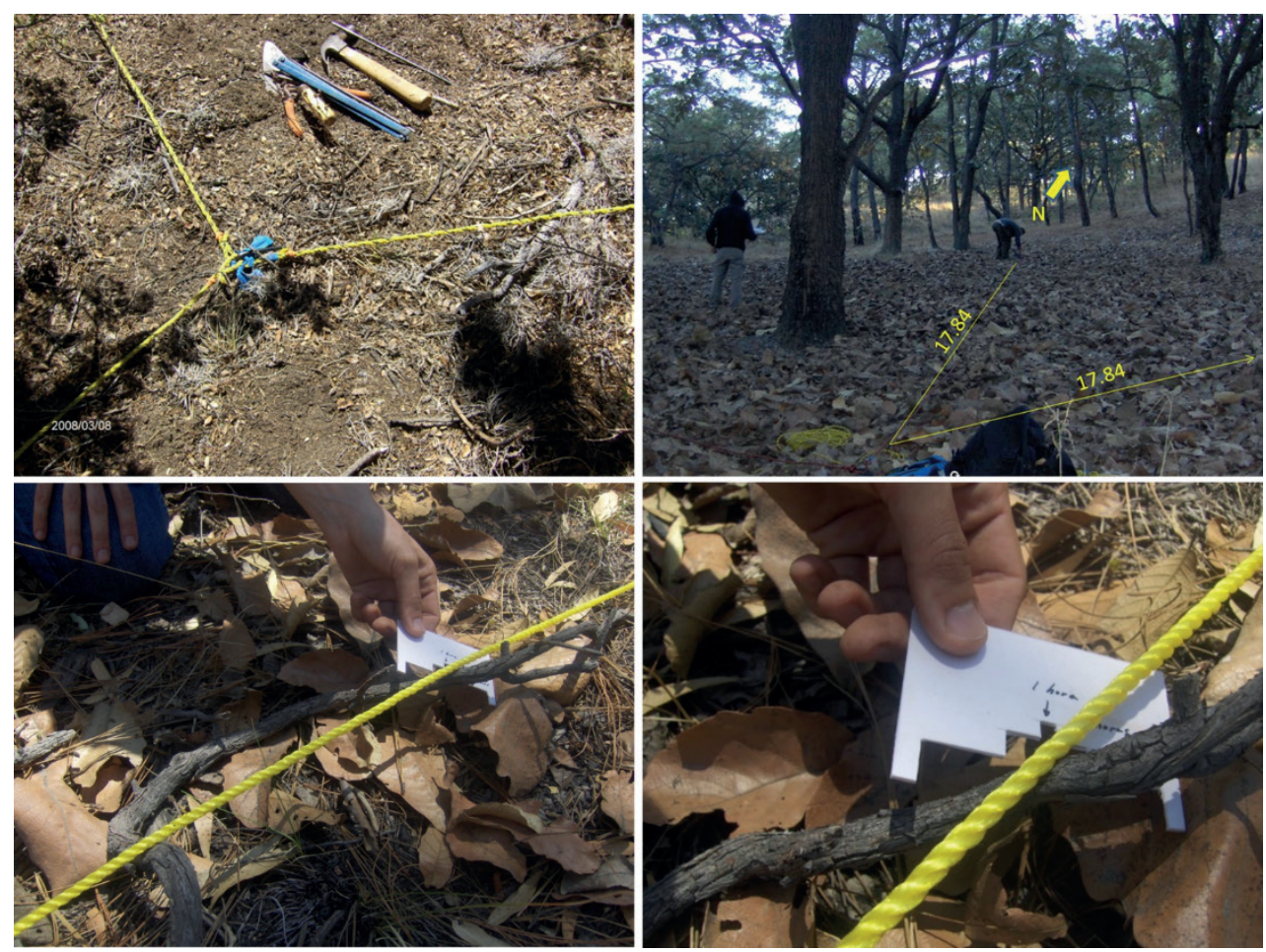

Figura 3. Establecimiento de transectos y conteo de material leñoso caído, basado en la metodología de intersecciones planares (Fotografías: J. Germán Flores G.).
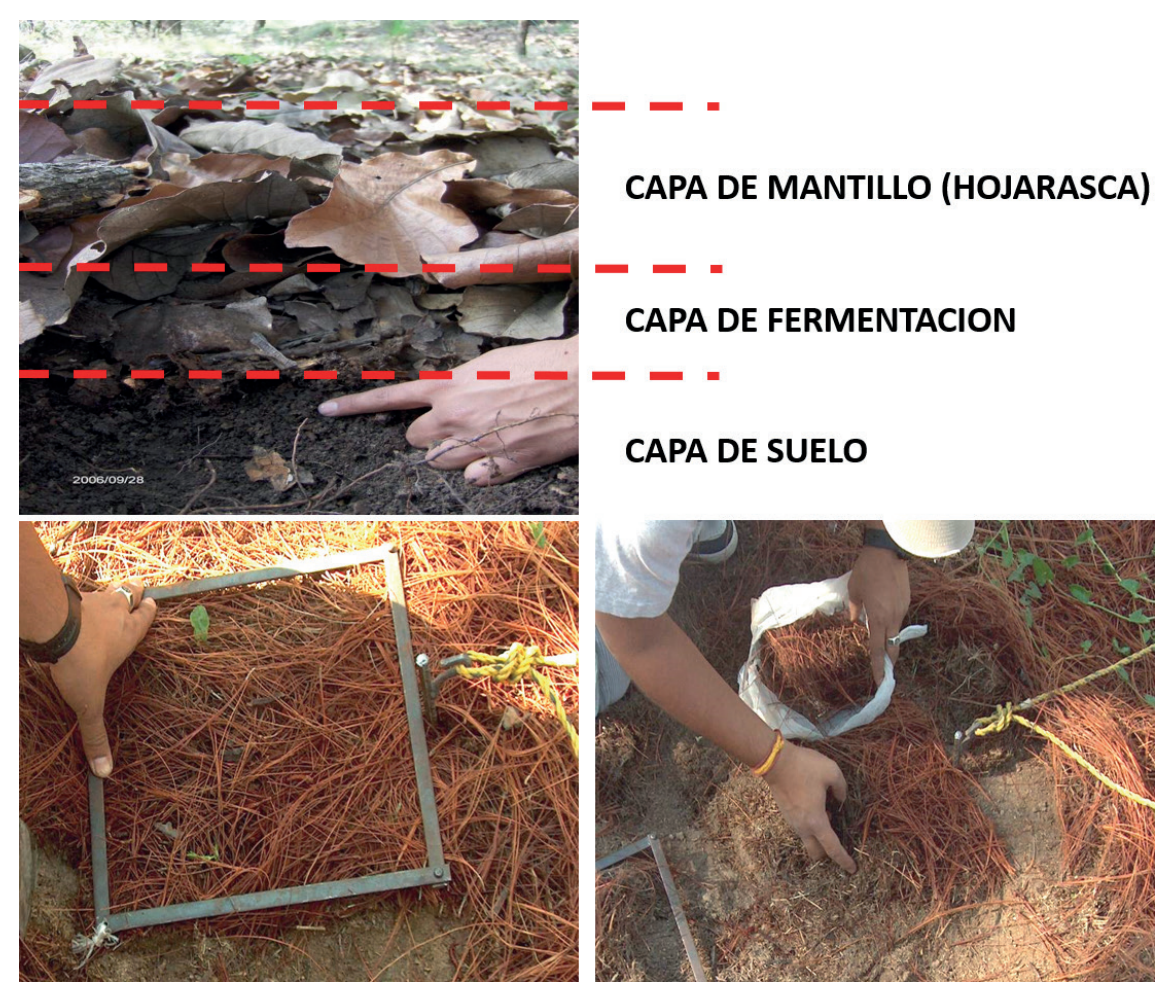

Figura 4. Separación de la capa de hojarasca y fermentación y cuadro de $30 \mathrm{~cm} \times 30 \mathrm{~cm}$ para la toma de muestras de estos combustibles finos (Fotografías: J. Germán Flores G.). 


\section{RESULTADOS}

\section{Porcentaje de contenido de carbono}

Como se ha mencionado, generalizar las evaluaciones de $\mathrm{C}$ de los combustibles forestales en pocos componentes puede implicar la definición de un valor alto de incertidumbre (Kurz et al., 2016), debido a dos aspectos importantes: 1) varias clasificaciones de combustibles pueden desglosarse en una serie de componentes, por ejemplo, la biomasa aérea comprende follaje, fuste, ramas, arbustos, hierbas, pastos, etc. y 2) los diferentes combustibles forestales pueden contener diferentes proporciones de $\mathrm{C}$ (Avendaño, Acosta, Carrillo y Etchevers, 2009). En relación con esto último, la mayoría de los trabajos que han evaluado el contenido de $\mathrm{C}$ en combustibles forestales, optaron por usar un porcentaje de contenido de $\mathrm{C}$ respecto a la masa (peso seco) de 0.50, sin importar de qué tipo de combustibles se trata, lo cual, en sí, ya implica cierto valor de incertidumbre (Kurz et al., 2016). En la figura 5 se compara el porcentaje de contenido de $\mathrm{C}$ usado para estimar el contenido de C, tanto en MLC como en M. Se resalta que el mayor contenido de $\mathrm{C}$ se presenta en los combustibles leñosos firmes (no podridos) mayores a $7.5 \mathrm{~cm}$, donde se encuentra un máximo de 0.669 (Flores, inédito); mientras que, en promedio, las proporciones menores se registran para hojarasca. En general, el porcentaje mínimo fue 0.475. De acuerdo con el rango de variación, es importante considerar que los porcentajes se usan para hacer estimaciones de almacenamiento de $\mathrm{C}$ a varias escalas (Anaya, Chuvieco y Palacios, 2009), por lo que estas estimaciones, de acuerdo con los trabajos analizados, podrían implicar una variación de $\pm 10.33 \%$ respecto de la media. Lo anterior involucra una incertidumbre importante, misma que se magnifica a medida que se escalan (árbolsitio-hectárea-rodal-región-estado-país) las estimaciones, por lo que debe definirse a corto plazo un catálogo de porcentaje de contenido de C para los ecosistemas de México y por tipo de combustibles.

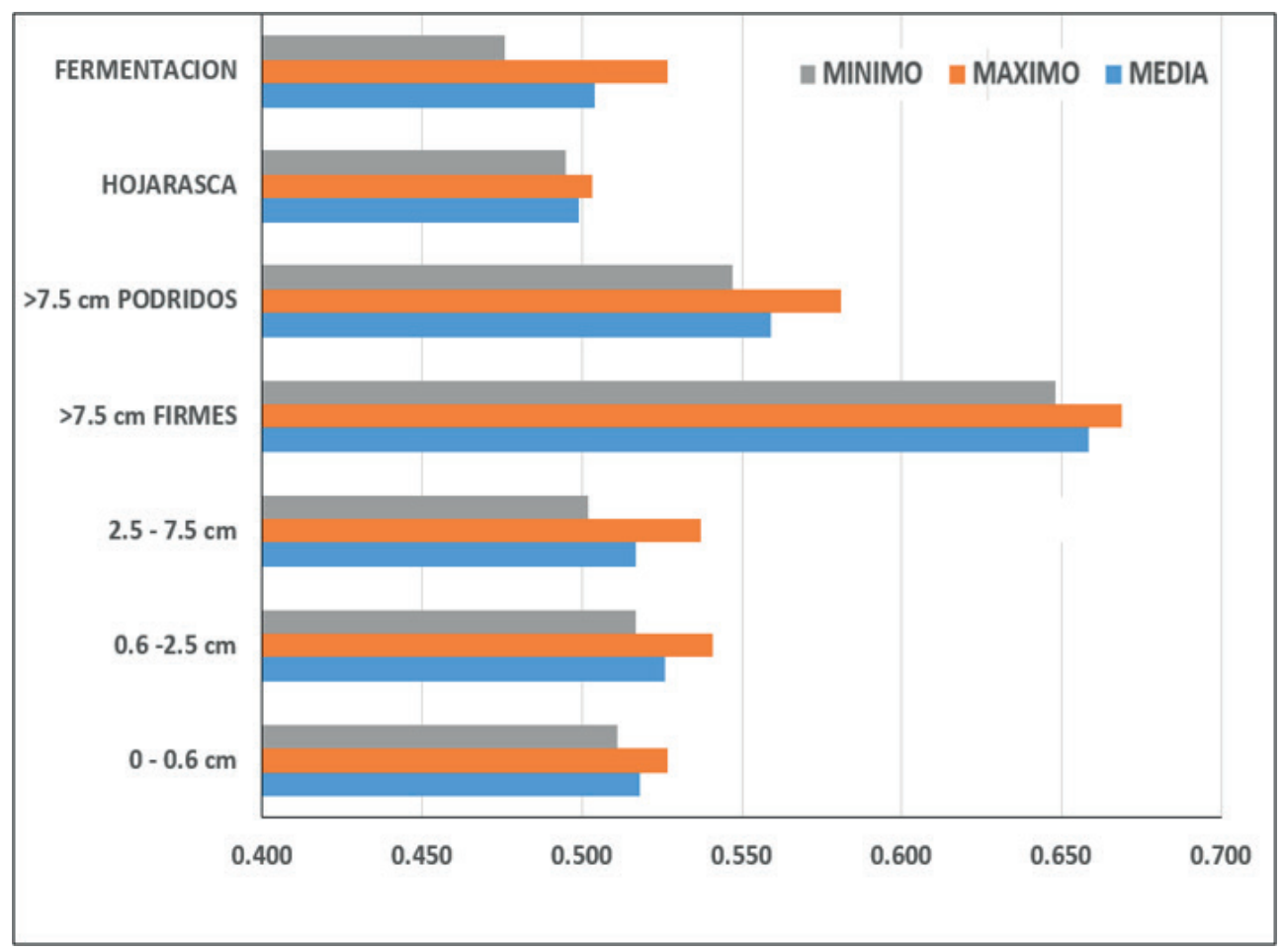

Figura 5. Porcentajes de contenido de carbono respecto a la masa (peso seco) usados en trabajos enfocados en la estimación de C de combustibles forestales en México (Flores, inédito). 


\section{Contenido de carbono}

Aunque existen muchos trabajos sobre la evaluación de cargas de combustibles forestales, son pocos los que se enfocan en la evaluación de C (Navarrete, 2006). Al respecto, en la tabla 3 se presenta un panorama en México, el análisis se basó en la PI, haciendo una estimación promedio de las evaluaciones de C para M y MLC (publicaciones del PMC: Paz y Cuevas, 2012; Paz y Wong, 2015; Paz, Wong, Bazan y Saynes, 2014; Paz, Wong y Torres, 2015; Wong y Paz, 2016). La mayor parte de los estudios donde se ha evaluado M y MLC se ubican en las regiones templadas del país, cuyo principal propósito es la estimación de cargas de combustibles $\left(\mathrm{Mg} \mathrm{ha}^{-1}\right)$, con base en lo que se calculan los contenidos de C correspondientes. De esta manera se tienen trabajos en varios ecosistemas, como: bosques mixtos de coníferas (Stephens, Fry, Franco, Collins y Moghaddas, 2007), bosques montanos de niebla (Álvarez, García, Krasilnikov y García, 2013), bosque mesófilo de montaña (Álvarez, 2010), bosques mixtos (Naranjo, 2014), entre otros. Estas estimaciones se han hecho también en sistemas agroforestales con predominio de cultivo de café (Rojas, 2014), o en forma comparativa entre sistemas forestales y agrícolas (Martínez, 2010), además de zonas urbanas (Rodríguez y Sierra, 1995). Específicamente, se han desarrollado estimaciones de M y MLC en bosque de pino, encino y oyamel (Álvarez, 2010; Bautista, Treviño, Návar, Aguirre y Cantú, 2005; Nájera y Hernández, 2007; Naranjo, 2014; Navarrete, 2006; Rojas, 2014; Stephens et al., 2007; Xelhuantzi et al., 2011).

Como se ha mencionado, las estimaciones de $\mathrm{M}$ y MLC, aunque se basan en técnicas generalizadas, no han seguido un proceso estandarizado, por lo que los resultados deben adaptarse para que puedan ser compatibles y comparables. De esta forma, primeramente se debe hacer una estimación de las cargas de combustibles, para lo cual la mayoría de los trabajos se basan en la técnica de intersecciones planares, propuesta por Brown (1974) y Flores y Omi (2003); aunque en algunos casos se han realizado adaptaciones, o modificaciones a esta técnica (Nájera y Hernandez, 2007; Naranjo, 2014; Rodríguez y Sierra, 1995). Estas adaptaciones se enfocan principal- mente en variaciones en la longitud de los transectos (Bautista et al., 2005; Orihuela et al., 2013; Rodríguez et al., 2011; Rodríguez y Sierra, 1995; Rojas, 2014; Venegas, 2013; Xelhuantzi et al., 2011). Otras estrategias de evaluación de cargas de combustibles que se han usado son: a) evaluaciones directas de las cargas de combustibles (Álvarez et al. 2013; Eaton, 2005); b) método de intersección planar adaptado (Aguirre, 2006); c) referencia a un muestreo jerárquico anidado (Álvarez et al. 2013); d) consideración de cinco grados de pudrición del material combustible (Eaton, 2005).

\section{DISCUSIÓN}

La forma en que se publican los resultados de las evaluaciones referentes al M y MLC son diversas. No obstante, considerando la estructura que se propone en la tabla 1 , se puede tratar de organizar esos resultados. De esta forma, la tabla 3 presenta una serie de estimaciones de $C$ en material leñoso y mantillo, para dos grandes regiones (bosques y selvas), pero es importante aclarar que las cifras corresponden a los promedios de las estimaciones publicadas en varios trabajos. Por ejemplo, la media que se señala no necesariamente corresponde a la media de las cifras máxima (máx) y mínima (min) de la tabla 3 , sino que corresponde a la media de todos los trabajos analizados. También se aclara que, debido a que en muchos casos solo se encontraron cargas de combustibles $\left(\mathrm{Mg} \mathrm{ha}^{-1}\right)$, se usó el valor de 0.5 como porcentaje de estimación del contenido C. Por otra parte, se indican variaciones en MLC debido a diferentes altitudes, donde combustibles entre $10.1 \mathrm{~cm}$ y $20 \mathrm{~cm}$ presentan un mayor contenido de $\mathrm{C}$, en general en la altitud de $1500 \mathrm{~m}$ snm (Fig. 6). Lo mismo sucede en el caso de los combustibles mayores a $20 \mathrm{~cm}$ (Álvarez et al. 2013). También se señalan cargas de combustibles para ecosistemas forestales específicos, con las cuales, considerando un porcentaje de 0.5 , se tienen las siguientes estimaciones de $\mathrm{Mg} \mathrm{ha}^{-1}$ de C (Rodríguez y Sierra, 1995): pino y latifoliadas $11.6 \mathrm{Mg} \mathrm{C}^{-1}$, oyamel y pino $13.6 \mathrm{Mg} \mathrm{C} \mathrm{ha}^{-1}$, oyamel de saneamiento $32.1 \mathrm{Mg} \mathrm{C} \mathrm{ha-1.} \mathrm{También} \mathrm{se} \mathrm{regis-}$ tran evaluaciones totales para selva (Fonseca, Rey y Alice, 2011; Hughes, Kauffman y Jaramillo, 1999; Romero, 
2008), plantaciones tropicales y manglar, donde el último autor señala el mayor contenido de C (124 Mg C ha-1).

En cuanto a la evaluación de combustibles en las regiones de selva existen pocos trabajos, y menos aun los que hagan evaluaciones específicas para M (hojarasca y fermentación) (Xelhuantzi et al., 2011); mientras que en otros ecosistemas solo se registran cifras totales. En cuanto a la capa de fermentación son pocos los trabajos enfocados en la evaluación de $\mathrm{C}$, resaltando la cifra que se encontró para manglar (20.92 $\left.\mathrm{Mg} \mathrm{C} \mathrm{ha}^{-1}\right)$.

\section{CONCLUSIONES}

En general, considerando los trabajos sobre los diferentes combustibles forestales englobados en la PI, se encontró muy poca información sobre las evaluaciones de C corres- pondientes al M y MLC, las cuales se han enfocado principalmente a ecosistemas templados, seguido de ecosistemas tropicales. Más aún, los trabajos son menos si se particulariza en algunos ecosistemas, como bosque de pino, bosque de encino, bosque de oyamel, selva baja, selva mediana, manglares, entre otros.

La mayoría de los trabajos de evaluación de combustibles (cargas) se han realizado en ecosistemas templados. Sin embargo, no se ha seguido un proceso estandarizado, ya que en la mayoría de los casos se hicieron adaptaciones a la metodología de Brown et al. (1982), sin ofrecer la explicación de estas modificaciones. Sobre todo, desde la perspectiva estadística.

Considerando los diferentes tipos de combustibles de material leñoso, los contenidos de $\mathrm{C}$ en los combustibles

TABla 3. Contenido de C (Mg) por hectárea del mantillo y material leñoso de regiones forestales templadas y de selva en México.

\begin{tabular}{|c|c|c|c|c|c|c|c|c|c|}
\hline Condición & Estrato & Subestrato & Variable & $\begin{array}{l}\text { Selva } \\
\min \end{array}$ & $\begin{array}{l}\text { Selva } \\
\text { máx }\end{array}$ & $\begin{array}{l}\text { Selva } \\
\text { media }\end{array}$ & $\begin{array}{c}\text { Templado } \\
\text { mín }\end{array}$ & $\begin{array}{l}\text { Templado } \\
\text { máx }\end{array}$ & $\begin{array}{c}\text { Templado } \\
\text { media }\end{array}$ \\
\hline & & Fino & $0 \mathrm{~cm}-0.6 \mathrm{~cm}$ & 0.01 & 0.85 & 0.44 & 0.04 & 0.23 & 0.09 \\
\hline & & & $0.6 \mathrm{~cm}-2.5 \mathrm{~cm}$ & 0.03 & 3.00 & 1.80 & 0.01 & 4.32 & 1.36 \\
\hline & Material & Grueso & $2.5 \mathrm{~cm}-7.5 \mathrm{~cm}$ & 0.06 & 3.55 & 2.52 & 0.01 & 5.21 & 1.53 \\
\hline & leñoso & & $>7.5 \mathrm{~cm}$ firme & 0.01 & 7.90 & 3.75 & 0.15 & 13.28 & 3.64 \\
\hline & & & $>7.5 \mathrm{~cm}$ podrido & 0.02 & 7.50 & 3.23 & 0.01 & 8.78 & 4.39 \\
\hline & & Total & & 1.35 & 10.01 & 8.00 & 0.03 & 32.10 & 9.43 \\
\hline & & Coníferas & Hojas & & & & 0.05 & 9.05 & 4.96 \\
\hline & & & $\begin{array}{l}\text { Flores-frutos- } \\
\text { semillas }\end{array}$ & & & & 0.00 & 2.90 & 0.57 \\
\hline \multirow[t]{9}{*}{ Necromasa } & Mantillo & Latifoliadas & Hojas & 0.78 & 5.22 & 2.01 & & & \\
\hline & & & $\begin{array}{c}\text { Flores-frutos- } \\
\text { semillas }\end{array}$ & 0.00 & 0.83 & 0.13 & & & \\
\hline & & Total & & 0.78 & 6.04 & 4.25 & 4.00 & 19.70 & 6.91 \\
\hline & & & Hojarasca & 0.00 & 2.85 & 1.27 & 0.00 & 14.98 & 6.71 \\
\hline & & & Madera-corteza & & & & & & \\
\hline & Capa de & & Flores-frutos- & & & & & & \\
\hline & Fermentación & & semillas & & & & & & \\
\hline & & & Raíces & & & & & & \\
\hline & & Total & & 0.00 & 3.67 & 1.39 & 0.00 & 17.88 & 5.88 \\
\hline
\end{tabular}




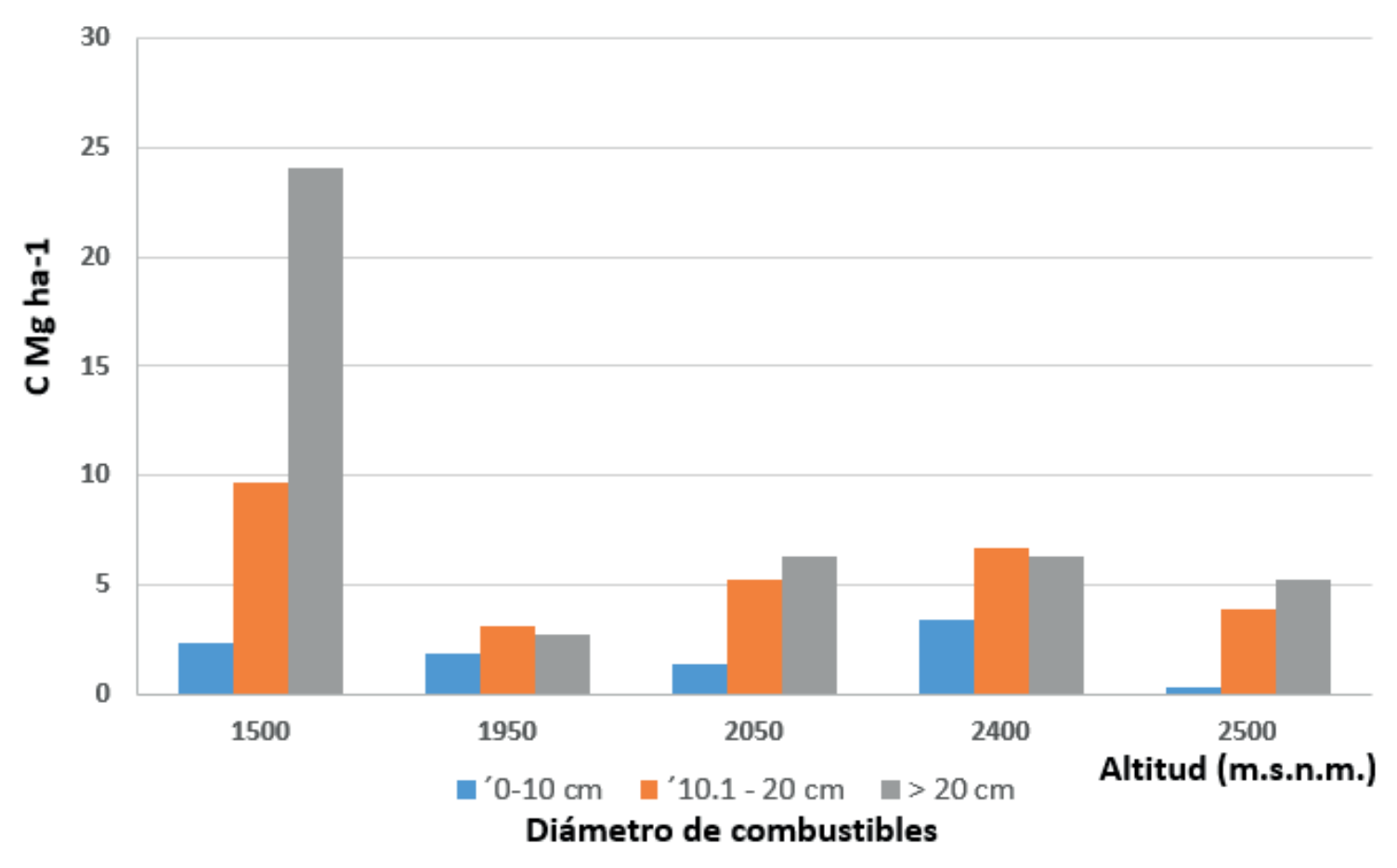

Figura 6. Variaciones en el contenido de C de combustibles leñosos, que corresponden a diferentes altitudes (m snm) (adaptado de Álvarez et al. 2013).

forestales fueron muy similares en sus promedios entre selvas y ecosistemas templados. Aunque, por otro lado, los contenidos máximos fueron mayores en los ecosistemas templados. Considerando los contenidos totales de C, los valores máximos son tres veces mayores en ecosistemas templados que en selvas. No obstante, los promedios de contenido son muy similares.

Los promedios máximos totales de las evaluaciones del contenido de $\mathrm{C}$ en el mantillo fueron tres veces mayores en ecosistemas templados, en relación con las selvas. Aunque, en la media de los valores totales el contenido de $\mathrm{C}$ en ecosistemas templados fue $50 \%$ mayor en selvas. En cuanto a la capa de fermentación, los promedios totales máximos en ecosistemas templados fueron más de cinco veces mayor que en selvas. Sin embargo, estas evaluaciones se enfocaron en la hojarasca en pudrición y no se encontraron trabajos que indiquen evaluaciones referentes a la madera, corteza, flores, frutos, semillas o raíces.
En las evaluaciones de los contenidos de $\mathrm{C}$ se ha generalizado el uso de un porcentaje de contenido de $\mathrm{C}$ respecto a la masa (peso seco) promedio de $50 \%$; sin embargo, estas evaluaciones deben hacerse considerando que, por su condición, a cada tipo de combustible le corresponde un porcentaje específico. Lo cual, en la mayoría de los estudios analizados no se considera, realizándose evaluaciones con valores de contenido de $\mathrm{C}$ con un alto nivel de incertidumbre.

Las evaluaciones de C de M y MLC no se han realizado en forma protocolizada; es decir, siguiendo procedimientos de evaluación estandarizados. Por lo anterior, la información generada no es directamente comparable, ni compatible. Lo antepuesto propicia estimaciones dentro de intervalos muy amplios (es decir, alta variabilidad), que implican también altos niveles de incertidumbre.

En futuros trabajos se deberá caracterizar a los diferentes ecosistemas forestales considerando especies, estructura, densidad y edades, entre otros. De esta forma 
se podrán identificar los sitios en donde los combustibles forestales presenten mayores variaciones en los contenidos de C, lo cual determinaría en dónde se deberán de intensificar las investigaciones para estimar $\mathrm{C}$ en $\mathrm{M} \mathrm{y}$ MLC.

\section{REFERENCIAS}

Aguirre, D. (2006). Servicios Ambientales: Captura de carbono en sistemas de café bajo sombra en Chiapas, México. (Tesis de maestría). Universidad Autónoma Chapingo, Chapingo, Estado de México.

Álvarez, A. (2010). Evaluación de la captura y estabilidad del carbono en un ecosistema de bosque mesófilo de montaña a lo largo del gradiente altitudinal, en la Sierra norte de Oaxaca. (Tesis doctoral). Universidad Nacional Autónoma de México, CDMX, México.

Álvarez, A. G., García, N.E., Krasilnikov, P. \& García, F. (2013). Almacenes de carbono en bosques montanos de niebla de la sierra norte de Oaxaca, México. Agrociencia, 47(2), 171-180.

Anaya, J. A., Chuvieco, E., \& Palacios, A. (2009). Aboveground biomass assessment in Colombia: A remote sensing approach. Forest Ecology and Management, 257, 12371246. doi: 10.1016/j.foreco.2008.11.016

Avendaño, H. D., Acosta, M. F., Carrillo, J., \& Etchevers J. (2009). Estimación de biomasa y carbono en un bosque de Abies religiosa. Revista Fitotecnia Mexicana, 32(3), 233 238.

Barrales, E., Carrasco, E. F., Etchevers, J. D., Hidalgo, C., Jiménez, D. C. \& Saynes, V. (2012). Carbono orgánico y emisión de carbono en mantillo y capa de fermentación. En F. Paz, \& R. Cuevas (Eds.), Estado Actual del Conocimiento del Ciclo del Carbono y sus Interacciones en México: Síntesis a 2011. Serie Síntesis Nacionales (pp. 368-373). Texcoco, Estado de México, México: Programa Mexicano del Carbono - Universidad Autónoma del Estado de México - Instituto Nacional de Ecología.

Bautista, J. A., Treviño, E. J., Návar, J. J., Aguirre, O. A. \& Cantú, I. (2005). Caracterización de combustibles leñosos en el ejido Pueblo Nuevo, Durango. Revista Chapingo, Serie ciencias forestales y del ambiente, 11(1), 51-56.
Brown, J. K. (1974). Handbook for inventorying downed woody material. USDA Forest Service General Technical Report, (16), 1-32.

Brown, J. K., Oberheu, R. D. \& Johnston, C. M. (1982). Handbook for Inventorying Surface Fuels and Biomass in the Interior West. USDA Forest Service General Technical Report (129), 1-52.

Chávez, A. A., Rubio, E., Flores, J. G., Luna, M., Flores, H. E., Ruíz, J. A., Ramírez, G. \& Carmona, J. X. (2014). Caracterización y clasificación de camas de combustibles prioritarios en México para planificar el manejo del fuego. Fundamentos técnicos y metodológicos. Libro Técnico Núm. 1. Tepatitlán de Morelos Jalisco, México: Instituto Nacional de Investigaciones Forestales, Agrícolas y Pecuarias.

Comisión Nacional Forestal [Conafor]. (2016). Inventario nacional forestal y de suelos. Procedimientos de muestreo Versión 15.8. México: Comisión Nacional Forestal.

Crutzen, P. J. \& Andreae, M. O. (1990). Biomass burning in the tropics: impact on atmospheric chemistry and biogeochemical cycles. Science, 250(4988), 1669-1678.

Cruz, G., Escalante, K., González, D., Guerra, E. \& Etchevers, J. (2012). Inventario de carbono en ambientes ribereños de montaña. En F. Paz, \& R. Cuevas (Eds.), Estado actual del conocimiento del ciclo del carbono y sus interacciones en México: Síntesis a 2011. Serie Síntesis Nacionales (pp. 195-200). Texcoco, Estado de México, México: Programa Mexicano del Carbono - Universidad Autónoma del Estado de México - Instituto Nacional de Ecología.

Eaton, J. (2005). Woody debris and the carbon budget of secondary forests in the southern Yucatán peninsular region (Thesis Master of Science). University of Virginia, Charlottesville, Virginia, USA.

Eggleston, H. S., Buendia, L., Miwa, K., Ngara, T. \& Tanabe, K. (eds.). (2006). Agriculture, forestry and other land use IPCC Guidelines for National Greenhouse Gas Inventories. Hayama, Japan: The Intergovernmental Panel on Climate Change.

Flores, J. G. \& Moreno, D. A. (2005). Modelaje espacial de la influencia de combustibles forestales sobre la regeneración natural de un bosque perturbado. Agrociencia, 39(3), 339-349. 
Flores, J. G. \& Omi, P. (2003). Mapping forest fuels for spatial fire behavior simulations using geomatic strategies. Agrociencia, 37(1), 65-72.

Flores, J. G. (Inédito). Porcentajes de contenido de carbono para estimar el contenido de carbono en combustibles forestales de México.

Fonseca, W., Rey, J. M. \& Alice, F. (2011). Carbon accumulation in the biomass and soil of different aged secondary forests in the humid tropics of Costa Rica. Forest Ecology and Management, 262(8), 1400-1408. doi:10.1016/j. foreco.2011.06.036

Fosberg, M. A., \& Deeming, J. E. (1971). Derivation of the 1and 10-hour time lag fuel moisture calculations for firedanger rating. USDA Forest Service Research Note RM-(207), 1-8.

Hughes, R. F., Kauffman, J. B., \& Jaramillo, V. J. (1999). Biomass, carbon, and nutrient dynamics of secondary forests in a humid tropical region of México. Ecology, 80(6), 1892-1907.

Hunter, M. L. (1990). Wildlife, forest, and forestry. principles of managing forests for biological diversity. New Jersey, USA: Prentice Hall.

Kurz, W. A., Birdsey, R. A., Mascorro, V. S., Greenberg, D., Dai, Z., Olguin, M. \& Colditz, R. (2016). Modelización $y$ evaluación integrada de la dinámica del carbono forestal en América del Norte: herramientas para monitorear, registrar en informes y proyectar emisiones $y$ remociones de gases de efecto invernadero en el sector forestal. Montreal: Comisión para la Cooperación Ambiental.

Maldonado, V., Paz, F., Olguín, M., \& de Jong, B. (2012). Caracterización de la cama de combustibles en los diferentes ecosistemas forestales mexicanos: elementos para la estimación de las emisiones de gases de efecto invernadero derivadas de los incendios. En F. Paz, \& R. Cuevas (Eds.), Estado Actual del Conocimiento del Ciclo del Carbono y sus Interacciones en México: Síntesis a 2011. Serie Síntesis Nacionales (pp. 417-421). Texcoco, Estado de México, México: Programa Mexicano del Carbono Universidad Autónoma del Estado de México - Instituto Nacional de Ecología.
Martínez, V. (2010). Almacenamiento de carbono en los sistemas forestales y agrícolas de la falda oeste del cerro Tláloc. (Tesis de maestría). Universidad Autónoma Chapingo, Chapingo, Estado de México.

Morfín, J. E., Jardel, E. J., Alvarado, E. C. \& Michel, J. M. (2012). Caracterización y Cuantificación de Combustibles Forestales. Universidad de Guadalajara, Jalisco, México: Editorial Universitaria.

Nájera, J. A. \& Hernández, E. H. (2007). Estimación de la carga de combustibles forestales en un bosque coetáneo de la región de El Salto, Durango. Sitio Argentino de Producción Animal, 1-4.

Naranjo, E. (2014). Evaluación de la carga de combustibles forestales en un bosque mixto de la sierra de Coyuca de Benitez, Estado de Guerrero, México. (Tesis de licenciatura). Instituto Tecnológico de Costa Rica, Cartago, Costa Rica.

Navarrete, J. (2006). Estimación del contenido de carbono en la biomasa leñosa muerta para diferentes clases de cobertura vegetal y uso de suelo: el caso de la región Purépecha, Michoacán. (Tesis de maestría). Universidad Nacional Autónoma de México, Ciudad de México, México.

Orihuela, D. E., de Jong, B. H., Mendoza, V. J., Van der Wal, J., Paz, F., Soto, L. \& Flamenco, S. A. (2013). Carbon stocks and accumulation rates in tropical secondary forests at the scale of community, landscape and forest type. Agriculture, Ecosystems and Environment, 171, 72-84. doi: 10.1016/j.agee.2013.03.012

Ottmar, R., Sandberg, D., Riccardi, C. \& Prichard, S. J. (2007). An overview of the Fuel Characteristic Classification System-Quantifying, classifying, and creating fuelbeds for resource planning. Canadian Journal of Forest Research, 37(12), 2383-2393. doi: 10.1 139/X07-077

Paz, F. \& Cuevas, R. (eds.). (2012). Estado Actual del Conocimiento del Ciclo del Carbono y sus Interacciones en México: Síntesis a 2011. Serie Sintesis Nacionales. Texcoco, Estado de México, México: Programa Mexicano del Carbono - Universidad Autónoma del Estado de México - Instituto Nacional de Ecología.

Paz, F. \& Wong, J. (eds.). (2015). Estado Actual del Conocimiento del Ciclo del Carbono y sus Interacciones en 
México: Síntesis a 2014. Serie Sintesis Nacionales. Texcoco, Estado de México, México: Programa Mexicano del Carbono - Centro de Investigación y Estudios Avanzados del Instituto Politécnico Nacional- Centro de Investigación y Asistencia en Tecnología y Diseño del Estado de Jalisco.

Paz, F., Wong, J., Bazan, M. \& Saynes, V. (eds.). (2014). Estado Actual del Conocimiento del Ciclo del Carbono y sus Interacciones en México: Síntesis a 2013. Serie Síntesis Nacionales. Texcoco, Estado de México, México: Programa Mexicano del Carbono - Colegio de Postgraduados - Universidad Autónoma de Chapingo Instituto Tecnológico y de Estudios Superiores de Monterrey.

Penman, J., Gytarsky, M., Hiraishi, T., Krug, T., Kruger, D., Pipatti, R., Buendia, L., Miwa, K., Ngara, T., Tanabe, K. \& Wagner, F. (Eds.). (2003). Good practice guidance for land use, land-use change and forestry. IPCC National Greenhouse Gas Inventories Programme. Japan: Intergovernmental Panel on Climate Change.

Prichard, S. J., Sandberg, D. V., Ottmar, R. D., Eberhardt, E., Andreu, A., Eagle, P. \& Swedin, K. (2013). Fuel Characteristic Classification System Version 3.0: Technical Documentation. General Technical Report PNW-GTR (887), 1-79.

Programa Mexicano del Carbono [PMC]. (2011). Manual de campo para el inventario estatal cuantitativo. México: Programa Mexicano del Carbono.

Pyne, S. J., Andrews, P. L. \& Laven, R. D. (1996). Introduction to wildland fire. New York, USA: John Wiley and Sons.

Rodríguez, D. A., Hubert, T., Cíntora, G. C., Contreras, A. R. \& de la Rosa, V. A. (2011). Modelaje del peligro de incendio forestal en las zonas afectadas por el huracán Dean. Agrociencia, 45(5), 593-608.

Rodríguez, D. A. \& Sierra, A. (1995). Evaluación de los combustibles forestales en los bosques del Distrito Federal. Revista Ciencia Forestal en México, 20(77), 193-218.

Rojas, P. (2014). Evaluación de carbono en sistemas agroforestales de café (Coffea arabica L.) en Chocamán, Veracruz. (Tesis de licenciatura). Universidad Autónoma Chapingo, Chapingo, Estado de México.
Romero, L. (2008). Diversidad y almacenes de carbono y nitrógeno en bosques tropicales caducifolios secundarios de la región de Chamela, Jalisco, con diferentes historias de uso. (Tesis doctoral). Ciudad de México, México: Universidad Nacional Autónoma de México.

Simard, A. J. (1968). The moisture content of forest fuels. Part III: moisture content variations of fast responding fuels below the fibre saturation point. Canadian Forest Service Information Report FFX (16), 1-50.

Stephens, S. L., Fry, D. L., Franco, E., Collins B. M. \& Moghaddas, J. M. (2007). Coarse woody debris and canopy cover in an old-growth Jeffrey pine-mixed conifer forest from the Sierra San Pedro Martir, Mexico. Forest Ecology and Management, 240, 87-95. doi:10.1016/j. foreco.2006.12.012

Torres, J. A. (1994). Wood decomposition of Cyrilla racemiflora in a tropical montane forest. Biotropica, 26(2), $124-140$.

Tubiello, F. N., Cóndor G., R. D., Salvatore, M., Piersante, A., Federici, S., Ferrara, A., Rossi, S., Flammini, A., Cardenas, P., Biancalani, R., Jacobs, H., Prasula, P. \& Prosperi, P. (2015). Estimación de emisiones de gases de efecto invernadero en la agricultura. Un manual para abordar los requisitos de los datos para los países en desarrollo. Roma: Organización de las Naciones Unidas para la Alimentación y la Agricultura.

Venegas, S. (2013). Efecto del manejo forestal en el contenido de carbono en vegetación y suelo de acabuales de Calakmul, México. (Tesis de maestría). El Colegio de la Frontera Sur, Campeche, México.

Wong, J. \& Paz, F. (2016). Memorias de resúmenes cortos. En F. Paz. (presidente del Congreso), VII Simposio Internacional del Carbono en México. Simposio dirigido por el Programa Mexicano del Carbono, Pachuca de Soto, Hidalgo.

Woodale, C. W. \& Williams, M. S. (2005). Sampling protocol, estimation, and analysis procedures for the down woody materials indicator of the FIA program. USDA Forest Service General Technical Report NC (256), 1-47.

Xelhuantzi, J., Flores, J. G. \& Chávez, A. A. (2011). Análisis comparativo de cargas de combustibles en ecosistemas 
forestales afectados por incendios. Revista Mexicana de

Ciencias Forestales, 2(3), 37-52.

Manuscrito recibido el 22 de marzo de 2017

Aceptado el 27 de enero de 2018

Publicado el 26 de noviembre de 2018
Este documento se debe citar como:

Flores-Garnica, J. G., Wong-González, J. C., \& Paz-Pellat, F. (2018). Camas de combustibles forestales y carbono en México. Madera y Bosques,24(Núm. esp.), e2401893. doi: 10.21829/myb.2018.2401893 (c) (1) (2) Madera y Bosques por Instituto de Ecología, A.C. se disEY NC SA ción-No Comercial-Compartir Igual 4.0 Internacional. 